\title{
Alkylation Process
}

National Cancer Institute

\section{Source}

National Cancer Institute. Alkylation Process. NCI Thesaurus. Code C40468.

Alkylating Activity in biological systems is typically a process of covalent bonding in which a chemical substance replaces hydrogen atom(s) in biologically active molecules with alkyl radical(s), hindering proper function. DNA alkylation results in strand cross-linking and can inhibit DNA replication. 